\title{
Larynx Organ Preservation in Patients with Hypopharyngeal-Laryngeal Cancer
}

\author{
Salvatore Conticello ${ }^{1 *}$, Andrea Fulcheri ${ }^{1}$, Salvatore Aversa ${ }^{1}$, Gabriella Gorzegno ${ }^{2}$, Alessio Petrelli ${ }^{3}$, \\ Giuseppe Malinverni ${ }^{4}$, Simona Allis ${ }^{5}$, Pietro Gabriele ${ }^{4}$, Cristina Ondolo ${ }^{1}$, Maria Grazia Ruo Redda ${ }^{5}$ \\ ${ }^{1}$ Department of Otolaryngology, University of Turin, San Luigi Gonzaga Hospital, Turin, Italy \\ ${ }^{2}$ Department of Medical Oncology, University of Turin, San Luigi Gonzaga Hospital, Turin, Italy \\ ${ }^{3}$ Epidemiology Unit, Turin, Italy \\ ${ }^{4}$ Department of Radiotherapy, Institute for Cancer Research and Treatment, Turin, Italy \\ ${ }^{5}$ Radiation Oncology Unit, University of Turin, San Luigi Gonzaga Hospital, Turin, Italy \\ Email: *salvatore.conticello@unito.it
}

Received October 8, 2012; revised November 13, 2012; accepted November 21, 2012

\begin{abstract}
Object: The therapeutic options for advanced laryngeal-hypopharyngeal cancer have broadened in the last decades, in the attempt to cure the cancer sparing laryngeal functions and to improve quality of life (functional surgery, chemoradiotherapy, combined therapy). Methods: We propose a single-centre based retrospective study on the results of the treatment of larynx-hypopharynx cancer on the basis of the different therapies offered, focusing on advanced-stage cancers. Among 146 patients with laryngeal-hypopharyngeal cancer treated in the period 1999-2006, we focused on 64 patients with advanced stage resectable cancer. In the larynx cancer group $-n=40,32$ patients had surgery and 8 patients had CT-RT (refusal of laryngectomy or relative contraindications to surgery). In the hypopharynx cancer group$\mathrm{n}=24,16$ patients underwent surgery and 8 patients had CT-RT. The outcome measure considered has been overall survival. Results: Larynx cancer group. Overall survival: after surgery we observed a 3 -year survival of $62 \%$, and a 5 -year survival of $44 \%$, while after CT-RT we had a 3-year survival of $25 \%$ and a 5 -year survival of $12 \%$. Hypopharynx cancer group. Overall survival: surgery: 3-year survival: 40\%, 5-year survival 32\%; CT-RT: 3 -year survival: $50 \%$, 5-year survival: $34 \%$. Conclusion: The results emphasize the use of larynx-preserving approaches for appropriately selected patients without a compromise in survival; in our case series, surgery had better outcome than CT-RT in advanced-stage larynx cancer; whilst no significant differences were observed in the treatment of hypopharynx cancer.
\end{abstract}

Keywords: Advanced Laryngeal-Hypopharyngeal Cancer; Survival; Surgery; Combined Therapy; Chemoradiotherapy

\section{Introduction}

Cancer of the larynx is among the most common cancers of the upper aero-digestive tract and it is diagnosed in nearly 10,000 men and women in the United States every year [1]. In Europe about 52,000 new cases of larynx cancer are discovered every year [2]. Hypopharynx cancer is a less common disease, representing in the United States, along with cervical esophageal cancer, as much as $10 \%$ of the tumors of the superior aero-digestive tract, and less than $1 \%$ of all cancers [3]. In more than half of the cases the tumour at diagnosis is an early stage larynx cancer, the remaining being advanced stage according to the AJCC classification [4]. The prevalence of larynx cancer is in Italy 142 per 100,000 (271 per 100,000 in men, and 22 per 100,000 in women) [5].

The main therapeutic options in the treatment of hypopharynx-larynx carcinomas are surgery-radical, endo

${ }^{*}$ Corresponding author. scopic and open partial, but also radiotherapy (RT) and, more recently, a combination of chemotherapy (CT) and RT. While data on the therapeutic indications and results in the treatment of early-stage carcinomas are well consolidated, the management of advanced-stage carcinomas causes much more discussions, because of the radical changes in the last twenty years $[4,6]$, as a consequence of the increased knowledge in biology, pharmacology, surgery and technology.

We propose a retrospective study on the cases of locoregionally advanced-stage hypopharynx and larynx squamous cell carcinomas (SCCs) we have observed in the period 1999-2006 at the Otolaryngology Dept. of the University of Turin; the primary end point of the study was to compare the outcome after surgery and after chemoradiotherapy (CT-RT) protocols.

It is intended to be the contribution of a monocentric experience, differently from other multicentric experiences available, which are at risk for bias and heterogeneity 
among the patients from the different centres.

\section{Patients and Methods}

Data on laryngoscopy procedures from surgery registers of the Department of Otolaryngology of the University of Turin were analyzed to identify the diagnosis of laryngeal-hypopharyngeal cancer. The study has included only patients affected by SCCs, thus excluding the cases of mucoepidermoid carcinomas, adenoid cystic carcinomas and verrucous carcinomas, which have biological features, natural history and treatment different from SCC [7]. The period considered has been 1999-2006 when 146 patients were treated for hypopharynx or larynx cancer (see Table 1 for characteristics). In particular the sites of the tumors included glottis, supraglottis and hemilarynx for laryngeal cancers, piriform sinus and pharyngo-larynx for hypopharyngeal cancers.

Among them 66 were previously untreated stage III or IV hypopharynx/larynx cancer; 2 were excluded (interruption of CT for severe acute toxicity, supportive care only): the remaining 64 advanced-stage patients were considered for analysis.

The informed-consent procedures and the study design followed national and international guidelines [8,9] and were reviewed and approved by the investigational board.

The follow-up ranged between 3 and 8 years, with a mean follow-up period of 51 months in the larynx cancer group, and 54 months in the hypopharynx cancer group. The characteristics of the 64 cases of resectable advanced-stage laryngeal-hypopharyngeal cancer analyzed are presented in Table 2.

Table 1. Characteristics of the case series.

Characteristics of hypopharynx-larynx Cancer patients (n: 146, Age range: 37 - 86 years, median age: 64.5 years, M/F: 11/1)

\begin{tabular}{ccc}
\hline Site $^{1}$ & Grading [2] & Stage \\
\hline Glottis: 72 (49\%) & G1:31 $31 \%)$ & Stage 0: 4 (3\%) \\
Supraglottis: 38 (26\%) & G2: 79(54\%) & Stage I: 37 $(26 \%)$ \\
Hemilarynx: 6 (4\%) & G3: 32 (22\%) & Stage II: 34 (23\%) \\
Pyriform sinus: 19(13\%) & & Stage III: 25 (17\%) \\
Pharyngolarynx: 11(8\%) & & Stage IV: 41 (28\%) \\
& & No data: 5 (3\%)
\end{tabular}

STAGE III-IV SITE [3] (n: 64)

Glottis: $13(20 \%)$

Supraglottis: $23(36 \%)$

Hemilarynx: $4(6 \%)$

Pyriform sinus: $18(28 \%)$

Paryngo-larynx: $6(10 \%)$

Characteristics of the complete hypopharynx and larynx cancer case series observed in the period 1999-2006. "Note that the dictions "Pharyngolarynx" and "Hemilarynx" refer to neoplasms so extended at laryngeal or pharyngolaryngeal level that attribution to a precise site of origin was impossible; [2] The sum of the 3 groups does not reach the total numbers of patients, because histological grading is defined only in invasive cancer, and so the 4 cases of carcinoma in situ in our sample are apart from this count [3]; This subgroup includes only the advanced-stage cancer patients considered for analysis $(66-2$ excluded $=64$ patients $)$.
Table 2. Cancer staging in advanced-stage hypopharyngeallaryngeal cancer.

\begin{tabular}{|c|c|c|c|c|}
\hline \multicolumn{5}{|c|}{ Larynx } \\
\hline Stage & TNM & Surgery & CT-RT & Total \\
\hline \multirow{3}{*}{ III } & T3N0 & 12 & 4 & 16 \\
\hline & T3N1 & 3 & & 3 \\
\hline & $\mathrm{T} 3 \mathrm{~N} 2$ & 6 & 2 & 8 \\
\hline \multirow[t]{2}{*}{ IVa } & T4aN0 & 6 & 1 & 7 \\
\hline & T4aN2 & 3 & 1 & 4 \\
\hline $\mathrm{IVb}$ & T4aN3 & 1 & & 1 \\
\hline \multirow[t]{2}{*}{ IVc } & T2N0M1 & 1 & & 1 \\
\hline & & 32 & 8 & 40 \\
\hline \multicolumn{5}{|c|}{ Hypopharynx } \\
\hline Stage & TNM & Surgery & CT-RT & Total \\
\hline \multirow{7}{*}{ IVa } & T3N0 & 1 & 1 & 2 \\
\hline & $\mathrm{T} 3 \mathrm{~N} 1$ & 1 & 1 & 2 \\
\hline & $\mathrm{T} 2 \mathrm{~N} 2$ & 3 & 1 & 4 \\
\hline & $\mathrm{T} 3 \mathrm{~N} 2$ & 3 & 2 & 5 \\
\hline & T4aN0 & 1 & & 1 \\
\hline & T4aN1 & 1 & & 1 \\
\hline & T4aN2 & 5 & 2 & 7 \\
\hline $\mathrm{IVb}$ & T3N3 & & 1 & 1 \\
\hline \multirow[t]{2}{*}{ IVc } & T2N0M1 & 1 & & 1 \\
\hline & & 16 & 8 & 24 \\
\hline
\end{tabular}

Patients in the surgery group were treated, when possible, with organ preservation surgery (14/48), in particular supraglottic laryngectomy and supracricoid partiallaryngectomy (with crico-hyoido-epiglottopexy: CHEPor crico-hyoidopexy: CHP-); when functional therapy was not possible, they received total laringectomy (34/48), followed by adjuvant RT when indicated; in the CT-RT group, the patients had CT-RT organ preservation in most of the cases because they refused total laryngectomy; a limited number because of surgical or medical contraindications towards surgery.

Salvage surgery was offered if the treatment failed to obtain complete response: in particular a subtotal laryngectomy was then made in a patient, because of recurrence of disease, and a laterocervical neck dissection was mandatory in another patient because of appearing of disease at that level.

The most common chemoradiotherapy protocol was based on cisplatin -CDDP- and 5-fluorouracil: -5-FU(induction with CDDP $100 \mathrm{mg} / \mathrm{m}^{2}$ at day 1 and 5 -FU $1000 \mathrm{mg} / \mathrm{m}^{2}$ days 1 to 5 every 21 days $\times 2$; concurrent CT-RT with CDDP $30 \mathrm{mg} / \mathrm{m}^{2}$ weekly and RT $68.4-70.2$ 
Gy and conventional fractioning 1 fraction/day, 1.8 - 2 Gy/day, $\mathrm{n}=8$ ).

Rarely a less aggressive protocol (concurrent cisplatinCDDP- and RT) or a chemoradiotherapy carboplatin (CBDCA) and taxol-based were used (the first one CDDP $30 \mathrm{mg} / \mathrm{m}^{2}$ weekly + RT $64-70.2 \mathrm{~Gy} /$ conventional fractioning, $\mathrm{n}=4$; the latter induction with CBDCA area under the curve, AUC $=6$ at day 1 and taxol $175 \mathrm{mg} / \mathrm{m}^{2}$ at day 2 repeated every 21 days $\times 2$ and concurrent CT-RT with CBDCA (AUC = 2) and RT 68.4 - 70.2 Gy, conventional fractioning, $n=4)$.

All of the patients considered but one completed the chemoradiotherapy protocol: such patient was in the CDDP-5-FU group and stopped the CT just before the fulfillment of the CT for toxicity, but has been however considered in survival analysis.

For each patient, data were collected from hospital registers and from clinical-endoscopic evaluation. Data were analyzed after 2010, to have a follow-up of at least three years. The outcome measure considered has been overall survival, that has been calculated from date of diagnosis to date of death or date of the last follow-up; when it was not possible to clinically evaluate the patients, a phone survey and, in case, an interrogation of the General Registry Office were carried out.

\subsection{Statistical Analysis}

Two groups were created on the basis of the cancer localization: the first included patients with cancer of glottis, supraglottis and hemilarynx $(n=40)$, the second one tumors of piryform sinus and pharyngolarynx $(\mathrm{n}=24)$; subjects were classified on the basis of the first line therapy, i.e. surgery or chemoradiotherapy. To analyze the relation between treatment and mortality non parametric survival curves (estimated with Kaplan-Meier method) were plotted, and Cox models were estimated, using treatment as model covariate. Hazard ratios of Cox models were evaluated using surgery group as reference category. Risk proportionality was tested with the logcumulative hazard plot. The analysis was performed using PROC LIFETEST and PHREG of SAS System.

\section{Results}

The results of survival analysis and Cox models are reported in Table 3. In the larynx cancer group the observed median survival time has been 47.2 months in the surgery group and 18.6 months in the CT-RT group. The 3 -year survival probability was $62 \%$ (se: 0.09 ) after surgery and 25\% (se: 0.15 ) after CT-RT, while 5-year survival probability was respectively $44 \%$ (se: 0.09 ) and $12 \%$ (se: 0.12 ).

In the hypopharyngeal group we found the median survival has been 33.1 months in surgery group, 36.1 months in CT-RT group. The 3-year survival probability was $40 \%$ (se: 0.13 ) after surgery and 50\% (se: 0.18 ) after CT-RT, while 5-year survival probability was respectively $32 \%$ (se: 0.13 ) and $34 \%$ (se: 0.18 ).

Figures 1 and 2 show the survival curves for larynx and hypopharyngeal cancer. For the larynx cancer the survival probability for the surgey group was always higher than for the CT-RT group.

Table 3. Results of survival analysis and Cox model.

\begin{tabular}{cccc}
\hline & & Larynx & Hypopharynx \\
\hline \multirow{4}{*}{ Surgery } & Median Survival Time (Months) & 47.2 & 33.1 \\
& $\mathrm{P}(\mathrm{S} \geq 3$ Yrs) & $62 \%$ & $40 \%$ \\
& $\mathrm{SE}$ & 0.09 & 0.13 \\
& $\mathrm{P}(\mathrm{S} \geq 5$ Yrs $)$ & $44 \%$ & $32 \%$ \\
& $\mathrm{SE}$ & 0.09 & 0.13 \\
CT-RT & Median Survival Time (Months) & 18.6 & 36.1 \\
& $\mathrm{P}(\mathrm{S} \geq 3$ Yrs) & $25 \%$ & $50 \%$ \\
& $\mathrm{SE}$ & 0.15 & 0.18 \\
Cox & $\mathrm{P}(\mathrm{S} \geq 5$ Yrs) & $12 \%$ & $34 \%$ \\
Model & $\mathrm{SE}$ & 0.12 & 0.18 \\
& $\mathrm{HR}$ & 2.98 & 0.75 \\
\hline
\end{tabular}

P: probability; S: survival; SE: standard error; HR: hazard ratio; 95\% CI: $95 \%$ confidence interval.

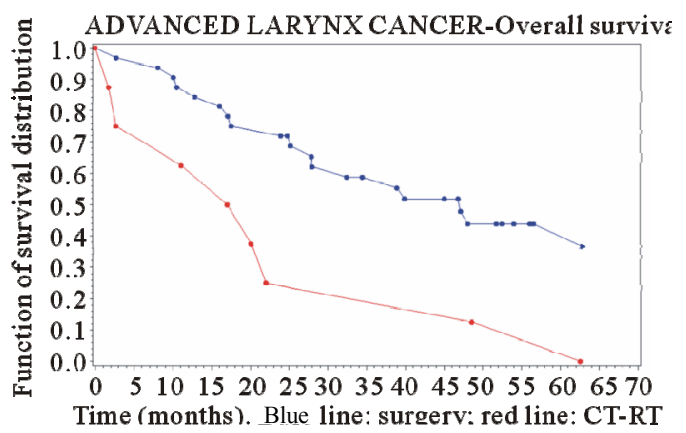

Figure 1. Survival curve in advanced-stage larynx cancer (time in months).

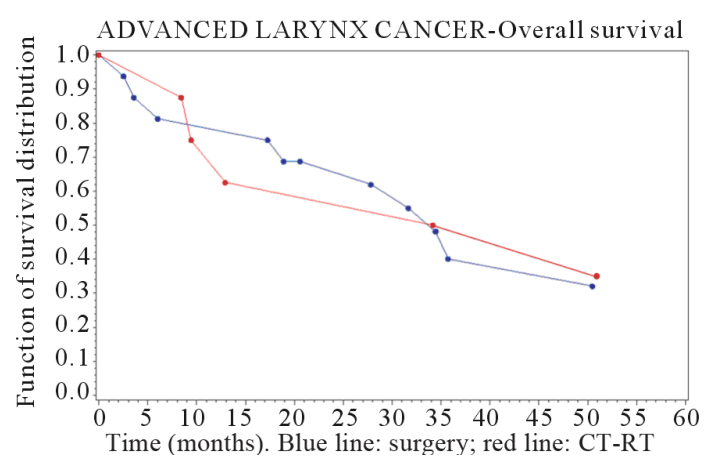

Figure 2. Survival curve in advanced-stage hypopharyngeal cancer (time in months). 
For the larynx cancer the risk of death was significantly higher for CT-RT group compared with surgery group (HR: 2.98; [1.28 - 6.97]), while a difference was found for hypopharyngeal cancer, with a slight better outcome after CT-RT than surgery, but it was not statistically relevant $(\mathrm{HR}=0.75[0.24-2.41])$.

\section{Discussion}

For many years, the only recommended treatment in advanced-stage carcinomas has been total laryngectomy followed by adjuvant RT. Many attempts have been made in order to avoid larynx mutilation in these tumours, both in surgery and in medical oncology and radiotherapy.

Transoral laser microsurgery has a limited role in advanced-stage cancer, even if it may be indicated in some T3 glottic or supraglottis tumor with normal arytenoid mobility [9].

Among many open partial laryngectomies, nowadays the most commonly employed are supraglottic laryngectomy, supracricoid partial laryngectomy (with CHEP or $\mathrm{CHP}$ ) that may be proposed in the treatment of $\mathrm{T} 3$ glottic or supraglottis tumor with arytenoid mobility and selected T4a glottic or supraglottis tumor without thyroid invasion. In selected cases subtotal laryngectomy with tracheo-hyoidopexy may be performed [9].

For what concerns non surgical organ preservation, the first study that explored this possibility has been a randomized trial on American Veterans (VALCSG), in which an induction CT was used to select patients who most likely would respond to RT, so that responders received RT while non-responders were surgically treated [9].

The next EORTC trial used a protocol similar to the VALCSG study, but enrolled patients with hypopharynx-larynx cancer, and not merely laryngeal tumors as the previous study [11].

RTOG 91-11 trial subsequently demonstrated that concomitant CT-RT could achieve higher larynx preservation rates than sequential schedule, even if the 5-year overall survival rates did not differ significantly [12].

These trials and the several that followed [13-16] demonstrated the possibility to achieve similar survival rates both using CT-RT or radical surgery, and certainly changed the standard of care in advanced-stage larynx cancer; these emerging data have not been accepted by everyone and most criticism arose, especially on the enrolling criteria of these studies and the possible bias of patients with an earlier stage disease in the experimental arm of these studies.

Besides, as Genden reports in his recent paper, there are not many reports evaluating the results of the different organ preservation and non-organ preservation approaches and data emerging from them are conflicting.
Moreover, it may seem that the quality of life in patients undergoing surgical and non-surgical therapies for advanced-stage laryngeal cancer is similarly compromised, although under different aspects [4].

In summary, among the organ preservation strategies organ preservation surgery in highly selected patients may achieve good oncological and functional results; chemoradiation protocols may obtain better oncological results in comparison with radiotherapy alone and have also effect on micrometastases, but there is higher acute toxicity than radiotherapy alone or surgery [17], and not always the preservation of the organ means the preservation of its function.

More recently, a multidisciplinary consensus panel presided by Lefebvre developed guidelines with recommendations for the conduct of clinical trials of larynx preservation in patients with locally advanced laryngeal and hypopharyngeal cancer [18]. The main key points indicated in the document are trial population features such as TNM classification, age and functional assessment. In particular, trial population should include patients with T2 or T3 laryngeal or hypopharyngeal SCC not considered for partial laryngectomyand should exclude those with laryngeal disfunction or age more than 70 years; moreover functional assessments should include speech and swallowing. The study also specified that primary endpoints should capture survival and function, and introduced the new end-point of laryngo-esophageal dysfunction-free survival.

Even if the present study was designed before such recent recommendations, we applied many criteria successively reported by Lefebvre et al. [18], considering reasonable differences, i.e. for what concerns T4 cancer treatment.

The case series presented is representative of the distribution of hypopharyngeal-laryngeal cancer for what concerns $\mathrm{M} / \mathrm{F}$ ratio, age, cases of early-stage disease at diagnosis, anatomical sites and sub-sites involved, and are aligned with data from literature [19].

Our data on larynx cancer show that results obtained with surgery are better than those obtained with CT-RT protocols. Furthermore, survival in the surgery arm of our study is similar to that from other Authors, while the results of CT-RT regimens observed in this study are worse than those published by other Authors [6]. Another important aspect is the fact that our retrospective case series resembles most suggestions recently indicated by Lefebvre et al. [18] in an important consensus panel on the modality to conduct larynx preservation clinical trials. These suggestions have been proposed in order to obtain studies and results more homogeneous and comparable than in the past decades.

Differently from the results we observed for larynx cancer, outcomes after surgery and CT-RT organ preservation 
in the hypopharynx cancer group were superimposable; such different responses to treatment between larynx and hypopharynx cancer are not surprising if we consider that cancer of the hypopharynx has generally more locoregional aggressiveness, more propensity for extensive microscopic regional infiltration and for an high incidence of distant metastasis, that better can be controlled by a "systemic treatment" as CT-RT is [20,21].

The study we present has been designed to analyze the results obtained in the clinical practice, and this makes impossible to randomize or to strictly balance our study population. The main limits of the present study are certainly the small numbers groups, especially for CT-RT group. We have to consider that larynx cancer is not very common, and so it is difficult to collect wide groups for a study. Besides, we have considered overall survival, and not disease-specific survival: this has brought us to overestimate mortality in the survival curves, nor we have presented other survival indicators, as recurrence rates, or recurrence-free survival. The only predictors of survival considered have been treatment. Retrospective reviews may have much weakness and may be at risk of bias, too. It is to underline the fact that a centre has the need to conform to the progresses in therapies- new drugs- and technologies: in the last ten years there have been many changes in CT-RT protocols, while modest ones in surgery and this is the reason why we don't have a "standard protocol" for the CT-RT, and slightly different CT-RT protocols are present in our retrospective analysis; the therapies offered however, were in accordance to the literature and national and international guidelines available for each period considered. Conversely, much more standard has been surgical indication and techniques.

The CT-RT treatment group included also patients who underwent salvage surgery for local or regional recurrence of the disease, and not only patients exclusively treated with CT-RT. Finally, we have not presented data on functional aspects of patients who underwent organ preservation-surgical or CT-RT- or radical surgery, nor on the presence of adverse events, such as dryness of mouth or fistulas: these data are missing in hospital registry and difficult to obtain in case of death, impossibility to find patients or their relatives.

The distance of our results in CT-RT organ preservation from those published by other Authors may have been conditioned by the limited numbers in the CT-RT arm and by the altered performance status of some of these patients.

As reported above, till now surgical therapy is the preferred treatment modality in hypopharynx-larynx carcinomas, with better results than non-surgical therapy in larynx cancer, and no differences in results in hypopharynxtumors. A scientific "CT-RT culture" in larynx cancer management is not widespread in Europe, where there is an evident surgical address maybe for the lack of a solid experience on CT-RT larynx organ preservation protocols and for the subsequent poor attention paid to this innovative therapeutic option at the moment of the therapy planning.

CT-RT organ preservation addressing has been discussed in a recent epidemiological study on patients with larynx cancer in which a reduction in survival was observed in the last twenty years, and it was correlated to the diffusion of CT-RT that prejudices of surgery [19]. On the other side, in the same period there was an improvement in survival in Europe, where there was a more frequent surgical approach.

A negative aspects of CT-RT organ preservation, is the loss of time before surgery in non-responders to induction $\mathrm{CT}$, and the presence of adverse events [22]. With regard to the first aspect, it is interesting the recent experience of Urba, which used a single CT course to select responders and non-responders, reducing the time to surgery in non-responders [23]. For what concerns adverse events, new biological drugs may improve the efficacy of CT agents, with a good toxicological profile [24-26].

The more positive results achieved by surgery in the treatment of advanced-stage larynx cancer, suggest us to prefer surgery to CT-RT, at least until we will have the possibility to select a group of patients that best will take advantage with CT-RT $[27,28]$. An important contribution may also come from recent genetics studies, with the identification of genetic predictors of chemoradiation resistance in advanced head and neck squamous cell carcinoma (HNSCC): the presence of different genomic profiles in sensitive and resistant HNSCCs may be valuable as predictive markers helpful at the moment of therapy planning [19].

As previously discussed, CT-RT organ preservation started in USA from 1980's, maybe also on the driving force of the worse surgical results obtained there than in Europe; in the last twenty years surgery made notable progresses both in the field of endoscopic surgery and open partial surgery (combined internal-external partial laryngectomy, subtotal laryngectomy with tracheoyoidopexy) [30,31].

Probably American otorhinolaryngologists, whose surgical address was less aggressive, turned their attention to the improvement of quality of life with non-surgical organ preservation; they thus obtained innovative results, but slow their surgical experience, in particular for what concerns supracricoid surgery and tracheoyoidopexy. Their use of surgery in case of failure of CT-RT, made them follow a way that does not allow a careful evaluation of the results obtained with surgery, since the results have been invalidated from multimodality therapy. On the contrary, European ENT specialists have not paid the right attention to $\mathrm{CT}$-RT organ preservation for their 
prevalent attention toward surgery [19-31].

We believe that CT-RT organ preservation has to be taken into account in the therapy planning, notwithstanding the possible presence of adverse events after CT-RT regimens; anyway we believe that patients with hypopharynx-larynx advanced cancer have to be treated, if possible, with surgical organ preservation, reserving CTRT organ preservation to patients in which there are reasons that exclude them from a surgical approach.

In perspective, for what concerns surgical organ preservation, we have to consider the advantages offered by the latest advancements in surgery (more aggressive laser endoscopic surgery, combined surgical techniques, tracheohyoidopexy). For what concerns CT-RT organ preservation the best protocol still need to be defined [32-34], even if we have to consider the better results obtained with tri-therapy regimes (cis-platin, 5-FU and docetaxel) in comparison to bi-therapy regimens, and the contribution of new cytotoxic agents (as taxane) that improve induction CT, and finally advancements in radiotherapy. All the different therapeutical options for larynx organ preservation have to be considered in order to preserve the functions and to improve the quality of life in patients with laryngeal cancer without impairing the survival; this requires special expertise, multidisciplinary management and a specialized support team.

In conclusion we think it would be appropriate to add to the term organ preservation an adjective that specify the procedure employed, i.e. surgical organ preservation or chemo-radiotherapic organ preservation; surgical organ preservation allows the preservation of part of the larynx with all its functions, while CT-RT organ preservation refers to a treatment without surgery, but only with chemo-radiotherapy that allows the preservation of all the larynx along with all its functions.

\section{Acknowledgements}

The authors are grateful to Prof. L. Dogliotti for his suggestions concerning medical oncology and to Prof. U. Ricardi for the permission to use data of the patients of our case series who underwent radiotherapy at his centre.

\section{REFERENCES}

[1] A. Jemal, R. Siegel, E. Ward, et al., "Cancer Statistics," CA: A Cancer Journal for Clinicians, Vol. 56, No. 2, 2006, pp. 106-130. doi:10.3322/canjclin.56.2.106

[2] J. Ferlay, F. Bray, P. Pisani, D. M. Parkin. "Cancer Incidence, Mortality and Prevalence Worldwide," IARC Cancer Base, No. 5, IARC Press, Lyon, 2001.

[3] D. G. Pfizer, K. S. Hun. "Cancer of the Hypopharynx and the Cervical Esophagus," In D. G. Pfizer and K. S. Hun, Eds., Head and Neck Cancer: A Multidisciplinary Approach, Lippincott Williams and Wilkins, Baltimore, 2004, pp. 404-454

[4] E. M. Genden, A. Ferlito, A. Rinaldo, et al., "Recent Changes in the Treatment of Patients with Advanced Laryngeal Cancer," Head Neck, Vol. 30, No. 1, 2008, pp. 103-110. doi:10.1002/hed.20715

[5] A. Micheli, S. Francisci, V. Krogh, A. G. Rossi and P. Crosignani, "Cancer Prevalence in Italian Cancer Registry Areas: The ITAPREVAL Study. ITAPREVAL Working Group," Tumori, Vol. 85, No. 5, 1999, pp. 309-369.

[6] J.-L. Lefebvre and D. D. Chevalier, "Neoplasie Della Laringe," EncyclMédChir (Editions Scientifiques et Médicales Elsevier SAS, Paris, Tutti i Diritti Riservati), Otorinolaringoiatria, 20-710-A-10, 2006.

[7] B. B. Koch, D. K. Trask, H. T. Hoffman, et al., "National Survey of Head and Neck Verrucous Carcinoma. Patterns of Presentation, Care and Outcome," Cancer, Vol. 92, No. 1, 2001, pp. 110-120. doi:10.1002/1097-0142(20010701)92:1<110::AID-CNCR 1298>3.0.CO;2-K

[8] F. L. Greene, D. L. Page, I. D. Fleming, et al., "AJCC Cancer Staging Handbook," Springer-Verlag, New York, 2002.

[9] M. De Vincentiis, et al., "Linee Guida Sul Cancro Della Laringe," Argomenti di Acta Otorhinolaryngologica Italica, Vol. 2, No. 4, 2008, pp. 33-49.

[10] The Department of Veterans Affairs Laryngeal Cancer Study Group, "Induction Chemotherapy Plus Radiation Compared with Surgery Plus Radiation in Patients with Advanced Laryngeal Cancer," The New England Journal of Medicine, Vol. 324, No. 24, 1991, pp. 1685-1690. doi:10.1056/NEJM199106133242402

[11] J.-L. Lefebvre, D. Chevalier, B. Luboinski, et al., "Larynx Preservation in Pyriform Sinus Cancer: Preliminary Results of a European Organization for Research and Treatment of Cancer Phase III Trial. EORTC Head and Neck Cancer Cooperative Group", Journal of the National Cancer Institute, Vol. 88, No. 13, 1996, pp. 890-899. doi:10.1093/jnci/88.13.890

[12] A. A. Forastiere, H. Goepfert, M. Maor, et al., "Concurrent Chemotherapy and Radiotherapy for Organ Preservation in Advanced Laryngeal Cancer", The New England Journal of Medicine, Vol. 349, No. 22, 2003, pp. 20912098. doi:10.1056/NEJMoa031317

[13] M. Machtay, D. I. Rosenthal, D. Hershock, et al., "Organ Preservation Therapy Using Induction Plus Concurrent Chemoradiation for Advanced Resectable Oropharyngeal Carcinoma: A University of Pennsylvania Phase II Trial," Journal of Clinical Oncology, Vol. 20, No. 19, 2002, pp. 3964-3971. doi:10.1200/JCO.2002.11.026

[14] E. E. Vokes, K. Stenson, F. R. Rosen, et al., "Weekly Carboplatin and Paclitaxel Followed by Concomitant Paclitaxel, Fluorouracil, and Hydroxyurea Chemoradiotherapy: Curative and Organ-Preserving Therapy for Advanced Head and Neck Cancer," Journal of Clinical Oncology, Vol. 21, No. 2, 2003, pp. 320-326. doi:10.1200/JCO.2003.06.006

[15] R. Haddad, R. B. Tishler, C. M. Norris, et al., "Docetaxel, Cisplatine, 5-Fluorouracile (TPF)-Based Induction Chemotherapy for Head and Neck Cancer and the Case for 
Sequential, Combined-Modality Treatment," The Oncologist, Vol. 8, No. 1, 2003, pp. 35-44.

doi: 10.1634/theoncologist.8-1-35

[16] Y. Pointreau, P. Garaud, S. Chapet, et al., "Randomized trial of Induction Chemotherapy with Cisplatin and 5Fluorouracil with or without Docetaxel for Larynx Preservation," Journal of the National Cancer Institute, Vol. 101, No. 7, 2009, pp. 498-506. doi:10.1093/jnci/djp007

[17] American Society of Clinical Oncology, D. G. Pfister, S. A. Laurie, G. S. Weinstein, et al., "American Society of Clinical Oncology Clinical Practice Guideline for the Use of Larynx-Preservation Strategies in the Treatment of Laryngeal Cancer," Journal of Clinical Oncology, Vol. 24, No. 22, 2006, pp. 3693-3704. doi:10.1200/JCO.2006.07.4559

[18] J.-L. Lefebvre and K. K. Ang, "Larynx Preservation Consensus Panel. Larynx Preservation Clinical Trial Design: Key Issues and Recommendations-A Consensus Panel Summary," Head \& Neck, Vol. 31, No. 4, 2009, pp. 429441.

[19] H. T. Hoffman, K. Porter, L. H. Karnell, et al., "Laryngeal Cancer in the United States: Changes in Demographics, Patterns of Care and Survival," Laryngoscope, Vol. 116, Suppl. 111, 2006, pp. 1-13. doi:10.1097/01.mlg.0000236095.97947.26

[20] R. S. Weber and H. Goepfert, "Cancer of the hypopharynx and cervical esophagus," In: E. N. Myers and J. Y. Suen, Eds., Cancer of the Head and Neck, 2nd Edition, Churchill Livingstone Inc., New York, 1989, pp. 509531.

[21] J.-L. Lefebvre and D. Chevalier, “Tumori Dell' Ipofaringe," EncyclMédChir (Editions Scientifiques et Médicales Elsevier SAS, Paris, Tutti i Diritti Riservati), Otorinolaringoiatria, 2005, p. 10.

[22] S. Conticello, C. Greco, S. Ferlito, et al., "La Patologia Iatrogenica Nelle Terapie Radianti," In: La Patologia Iatrogenica in ORL, Edizioni Minerva Medica, Torino, 1998.

[23] S. Urba, G. Wolf, A. Eisbruch, et al., "Single-Cycle Induction Chemotherapy Selects Patients with Advanced Latyngeal Cancer for Combined Chemoradiation: A New Treatment Paradigm," Journal of Clinical Oncology, Vol. 24, No. 4, 2006, pp. 593-598. doi:10.1200/JCO.2005.01.2047

[24] B. Burtness, M. A. Goldwasser, W. Flood, et al., "Phase III Randomized Trial of Cisplatin Plus Placebo Compared with Cisplatin Plus Cetuximab in Metastatic/Recurrent Head and Neck Cancer: An Eastern Cooperative Oncology Group Study," Journal of Clinical Oncology, Vol. 23, No. 34, 2005, pp. 8646-8654. doi:10.1200/JCO.2005.02.4646

[25] D. G. Pfister, Y. B. Su, D. H. Kraus, et al., "Concurrent Cetuximab, Cisplatin and Concomitant Boost Radiotherapy for Locoregionally Advanced, Squamous Cell Head and Neck Cancer: A Pilot Phase II Study of a New Combined-Modality Paradigm," Journal of Clinical Oncology, Vol. 24, No. 7, 2006, pp. 1072-1078. doi:10.1200/JCO.2004.00.1792

[26] J. A. Bonner, P. M. Harari, J. Giralt, N. Azarnia, et al., "Radiotherapy Plus Cetuximab for Squamous Cell Carcinoma of the Head and Neck," The New England Journal of Medicine, Vol. 354, No. 6, 2006, pp. 567-578. doi:10.1056/NEJMoa053422

[27] F. Dias, R. A. Lima, J. Kligerman, et al., "Therapeutic Options in Advanced Laryngeal Cancer: An Overview," ORL: Journal for Oto-Rhino-Laryngology, Head and Neck Surgery, Vol. 67, No. 6, 2005, pp. 311-318. doi:10.1159/000090040

[28] P. A. Pedruzzi, L. P. Kowalski, I. N. Nishimoto, et al., "Analysis of Prognostic Factors in Patients with Oropharyngeal Squamous Cell Carcinoma Treated with Radiotherapy Alone or in Combination with Systemic Chemotherapy," Archives of Otolaryngology, Head and Neck Surgery, Vol. 134, No. 11, 2008, pp. 1196-1204. doi:10.1001/archotol.134.11.1196

[29] G. B. Van den Broek, V. B. Wreesmann, M. W. van den Brekel, et al., "Genetic Abnormalities Associated with Chemoradiation Resistance of Head and Neck Squamous Cell Carcinoma," Clinical Cancer Research, Vol. 13, No. 15, 2007, pp. 4386-4391. doi:10.1158/1078-0432.CCR-06-2817

[30] S. Conticello, S. Biondi, S. Ferlito, "Indications and Results of Frontolateral Laryngectomy Using a Combined Endolaryngeal and External Approach," European Archives of Oto-Rhino-Laryngology, Vol. 256, No. 8, 1999, pp. 373-377. doi:10.1007/s004050050167

[31] G. Rizzotto, G. Succo, M. Lucioni, et al., "Subtotal Laryngectomy with Tracheohyoidopexy: A Possible Alternative to Total Laryngectomy," Laryngoscope, Vol. 116, No. 10, 2006, pp. 1907-1917. doi:10.1097/01.mlg.0000236085.85790.d5

[32] L. Lambert, B. Fortin, D. Soulières, L. Guertin, et al., "Organ Preservation with Concurrent Chemoradiation for Advanced Laryngeal Cancer: Are We Succeeding?" International Journal of Radiation Oncology, Biology andPhysics, Vol. 76, No. 2, 2010, pp. 398-402. doi:10.1016/j.ijrobp.2009.01.058

[33] J.-L. Lefebvre, "Larynx Preservation," Current Opinion in Oncology, Vol. 24, No. 3, 2012, pp. 218-222. doi:10.1097/CCO.0b013e3283523c95

[34] J. Ma, Y Liu, X. L. Huang, Z. Y. Zhang, et al., "Induction Chemotherapy Decreases the Rate of Distant Metastasis in Patients with Head and Neck Squamous Cell Carcinoma But Does Not Improve Survival or Locoregional Control: A Meta-Analysis," Oral Oncology, Vol. 48, No, 11, 2012, pp. 1076-1084

doi:10.1016/j.oraloncology.2012.06.014 